\title{
RESOLNRA: A new program for optimizing the achievable depth resolution of ion beam analysis methods
}

\author{
M. Mayer \\ Max-Planck-Institut für Plasmaphysik, EURATOM Association, Boltzmannstr. 2, \\ D-85748 Garching, Germany
}

\begin{abstract}
The code RESOLNRA for determining the experimental conditions (incident energy and angle) in order to achieve an optimal depth resolution was developed. RESOLNRA is based on the spectrum simulation code SIMNRA. RESOLNRA treats RBS, ERDA and NRA and includes the energy broadening contributions due to electronic energy loss straggling, geometrical straggling, multiple scattering, absorber foils and detector resolution. Additional constraints, such as minimum or maximum beam energies or tilt angles, or the requirement for a specific energy in a specific depth, can be taken into account.
\end{abstract}

Key words: Ion beam analysis, Computer simulation, Depth resolution, SIMNRA, RESOLNRA, RBS, ERDA, NRA, Straggling

PACS: 07.05.Tp, 82.80.Yc, 29.30.Ep

\section{Introduction}

For each element of a given sample there exists a unique combination of incident angle and incident energy, which achieves the best depth resolution for this element in a specific depth [1]. For many applications of ion beam analysis it is desirable to measure with the best possible depth resolution. However, many experimentalists still determine the conditions of their measurements by using simple rules-of-thumb. Consequently, many measurements do not achieve the depth resolution which would be possible, and they do not exploit the full potential of ion beam analysis methods.

This is mainly due to the fact, that it is difficult to find this optimum angle/energy combination. It depends on element and depth, and it is usually not possible to obtain the optimum depth resolution for several elements and/or 
a larger depth range simultaneously [1]. This requires either several optimized measurements, or a single measurement which achieves a reasonable compromise of depth resolutions over a larger depth range or for several elements, thus making the selection of the experimental conditions even more complicated.

The depth resolution is determined by the different energy broadening contributions due to energy loss straggling, geometrical straggling, multiple smallangle scattering of incident and exit ions, and the resolution of the detector [2,3]. The DEPTH code [2] allows to calculate the depth resolution as a function of sample depth, and it allows to determine the optimum angle/energy combination for a specific element and specific depth. However, for practical purposes this is usually insufficient: It is often important to know the precise shape of the depth-resolution minimum, and it is often important to take additional constraints (such as maximum or minimum incident energies or limitations of incident or exit angles) into account.

This paper describes the RESOLNRA code, which is based on the widely used SIMNRA spectrum simulation code [4,6]. The depth resolution is generally a function of depth, incident angle and incident energy for each isotope and each reaction ${ }^{1}$. RESOLNRA provides two- and three-dimensional cuts through this four-dimensional distribution: Resolution as function of depth for fixed angle and energy, resolution as function of incident energy for fixed depth and angle, resolution as function of incident angle for fixed depth and energy, and resolution as function of incident energy and angle for fixed depth as three-dimensional contour plots. The user interface was developed in order to allow a fast optimization of the experimental conditions, and the use of identical input files by RESOLNRA and SIMNRA offers the possibility of a rapid experimental design/experimental data evaluation cycle.

Energy distributions caused by geometrical straggling and multiple scattering get asymmetric for grazing incident or exit angles [5]. This is not taken into account by any analytical code up to now. RESOLNRA takes this asymmetry into account for geometrical straggling, thus providing a first step on the way to a more general treatment of asymmetric distributions.

\footnotetext{
$\overline{1}$ For the sake of readability we will use the term 'reaction' throughout this paper. A reaction may be backscattering, creation of a recoil, or a nuclear reaction emitting charged particles.
} 


\section{Calculation of depth resolution}

\subsection{Definition of the depth resolution}

An effective stopping power $S_{\text {eff }}$ can be defined by:

$$
S_{e f f}(x)=\left|\frac{d E}{d x}(x)\right|
$$

with $E$ the energy of a detected particle and $x$ the depth of origin, i.e. the depth where the reaction occurred. The depth resolution $\Delta x$ is then obtained from

$$
\Delta x(x)=\frac{\Delta E(x)}{S_{e f f}(x)},
$$

with $\Delta E(x)$ the energy spread of particles originating from the depth $x . \Delta E$ is the energy spread in full width at half maximum (FWHM), and correspondingly $\Delta x$ is the depth resolution in FWHM. See [2, Section 2] for a discussion of this definition.

\subsection{Depth resolution calculations by RESOLNRA}

RESOLNRA does not perform any energy-straggling or energy-loss calculations by itself. These calculations are performed by the SIMNRA code $[4,6]$, which is called through its OLE/COM interface. The SIMNRA-results are written to a log-file. RESOLNRA reads this log-file and calculates the effective stopping power and depth resolution from eqs. 1 and 2 by numerical differentiation.

The following energy broadening contributions are taken into account:

\subsubsection{Electronic energy-loss straggling}

Bohr's theory of electronic energy-loss straggling, the correction by Chu to Bohr's straggling [7,2], and the correction by Yang due to charge state fluctuations [8] are available and can be selected by the user. Propagation of straggling in thick layers (Tschalär effect) $[9,10,2]$ is always taken into account. An automatic step width control is used for the calculation of the Tschalär effect, thus providing a guaranteed numerical accuracy of energy-loss straggling calculations. The step width control is pre-set to an accuracy of $1 \%$ - a higher 
numerical accuracy does not make sense due to the general inaccuracies of straggling theories.

\subsubsection{Geometrical straggling}

Geometrical straggling is calculated according to [2]. SIMNRA 6.20 and higher uses the widths and heights of the incident beam spot and the detector diaphragm, and [2, eq. 30] is used for the parallel and perpendicular components. The energy distributions due to geometrical straggling get asymmetric for grazing incidence or exit paths. SIMNRA calculates the correct FWHM of these asymmetric distributions by calculating the energy of emerging particles with exit angles $\beta+\Delta \beta / 2$ and $\beta-\Delta \beta / 2$ at the target surface, where $\beta$ is the mean exit angle and $\Delta \beta$ the angular spread due to detector aperture and beam spot width or height.

\subsubsection{Multiple scattering}

Energy broadening due to multiple scattering on the incident and exit paths can be taken into account using the theory developed in $[2,3]$. The multiplescattering algorithms of DEPTH and SIMNRA are compared in [5] to the results of the Monte-Carlo code MCERD and the molecular dynamics code MDRANGE, and the limitations of the used analytical model are discussed. The energy distributions caused by multiple scattering are non-Gaussian with wider wings $[2,3]$. They are approximated by Gaussian functions with identical FWHM in SIMNRA.

\subsubsection{Absorber foil}

Arbitrary multi-layered foils can be placed in front of the detector. Additional electronic energy-loss straggling in the foil and the propagation of existing energy broadening is taken into account. While SIMNRA can calculate the effect of foil roughness on energy spectra, this feature is not implemented in RESOLNRA.

\subsubsection{Detector resolution}

Solid-state detectors with constant energy resolution $\Delta E$, electrostatic detectors with constant $\Delta E / E$, and time-of-flight detectors with constant $\Delta t / t$ (with $t$ the time-of-flight) can be used. 


\section{Results and discussion}

\section{$3.1 R B S$}

A thorough RBS-analysis of Co/Re multi-layers was performed by Barradas et al. in [11,12], the full set of spectra is shown in [13]. The samples consisted of 16 layers of rhenium with a nominal thickness of $5 \AA$, separated by cobalt layers with a thickness of $20 \AA$. A 40-50 $\AA$ thick impurity layer of carbon was found at the surface. The samples were analyzed with a $1 \mathrm{MeV}^{4} \mathrm{He}$ beam at a scattering angle of $160^{\circ}$ in Cornell geometry at incident angles of $45^{\circ}, 78^{\circ}, 80^{\circ}, 82^{\circ}, 83^{\circ}$ and $84^{\circ}$. The beam spot was $0.6 \times 0.2 \mathrm{~mm}^{2}$, with a detector aperture of $3 \mathrm{~mm}$ diameter at a distance of $70 \mathrm{~mm}$. As was already shown in [11,12], the layers showed some roughness which prevented to obtain the theoretically possible depth resolution. Nevertheless, the thorough and well designed measurements are an interesting test case for depth resolution optimizations. The surface roughness is neglected in the following.

The depth resolution for the isotope ${ }^{187}$ Re in the $1^{\text {st }}$ Re-layer is shown in Fig. 1 as a function of incident angle and incident energy. The optimum depth resolution of about $6 \times 10^{15}$ atoms $/ \mathrm{cm}^{2}$ is reached for incident energies of $800-4000 \mathrm{keV}$ and incident angles of $86-87^{\circ}$. For angles above $87^{\circ}$ the depth resolution degrades rapidly due to increasing geometrical straggling. The actual measurement was performed at $1000 \mathrm{keV} / 84^{\circ}$ and reaches a theoretical resolution of about $8 \times 10^{15}$ atoms $/ \mathrm{cm}^{2}$. Keeping the experimental difficulties at grazing angles in mind, this is as close to the theoretical optimum as is practically achievable.

The same as above, but for the $5^{\text {th }}$ Re-layer is shown in Fig. 2. The optimum depth resolution has now deteriorated to about $17 \times 10^{15}$ atoms $/ \mathrm{cm}^{2}$ due to the additional energy broadening contributions by energy loss straggling and multiple scattering, and the optimum depth resolution is now obtained for incident energies of $1400-4000 \mathrm{keV}$ and incident angles of $80-82^{\circ}$. The experiment reaches a theoretical resolution of about $20 \times 10^{15}$ atoms $/ \mathrm{cm}^{2}$ - this is close to the theoretical optimum, but does not fully reach it. A simultaneous change of incident angle and energy would have provided a better depth resolution than the actually performed change of incident angle at fixed energy, and would have reduced the overlap between the Re and Co signals.

The energy independence of the optimum depth resolution over a larger incident energy range, as shown in Fig. 2, may be surprising for many IBA practitioners: An often used rule-of-thumb for determining the incident energy is to set the energy of backscattered particles into the stopping-power maximum. The energy broadening contributions are shown in Fig. 3 for an 


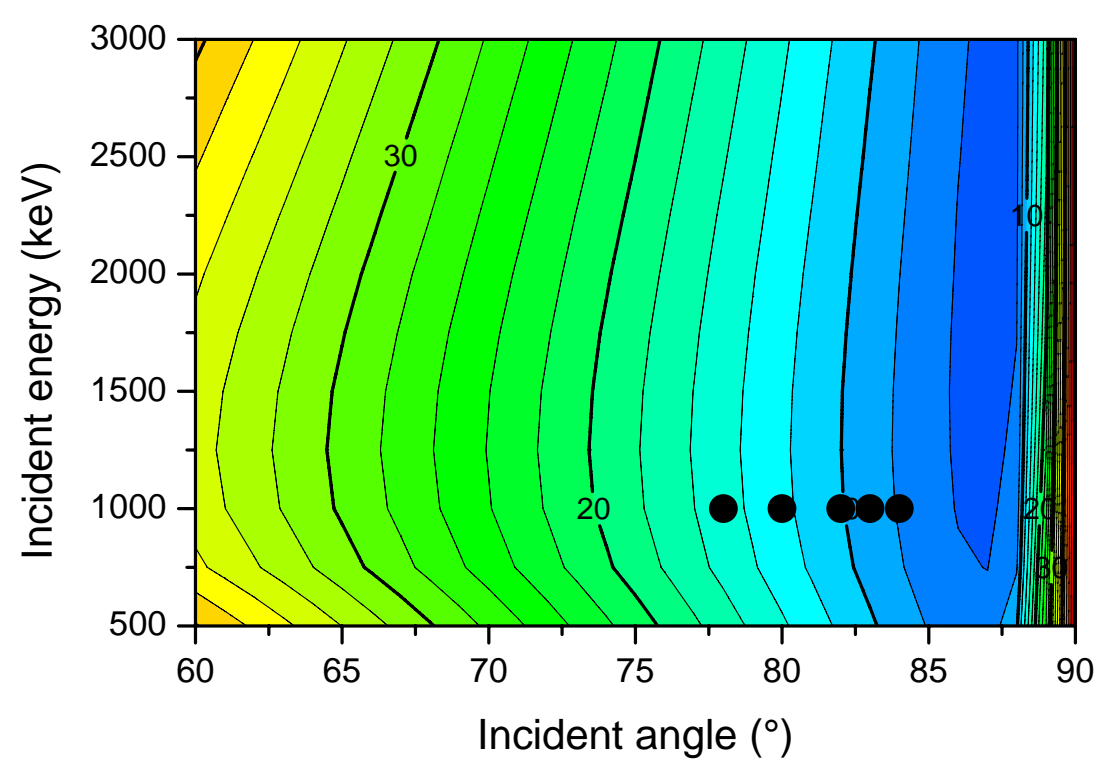

Fig. 1. Depth resolution for ${ }^{187} \operatorname{Re}$ (in $10^{15}$ atoms $/ \mathrm{cm}^{2}$ ) in the 1 st Re-layer as a function of incident angle and incident energy. Black dots indicate angle/energy combinations, at which measurements were performed in [11].

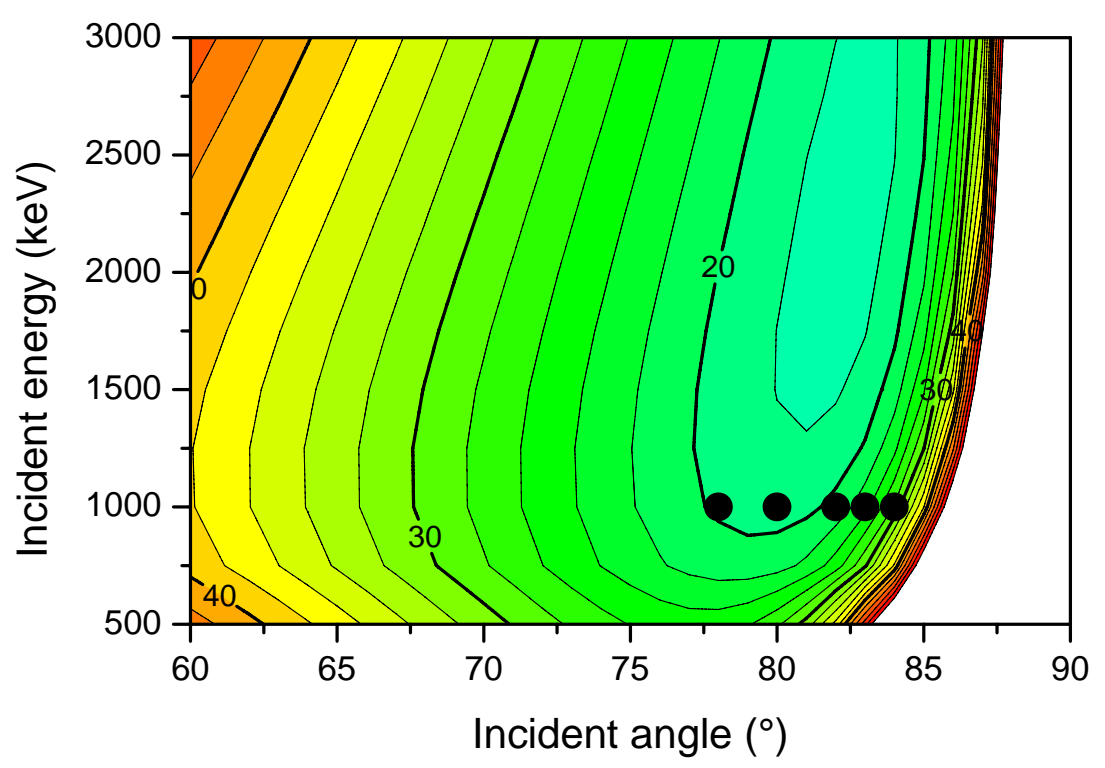

Fig. 2. Same as Fig. 1, but for the 5th Re-layer.

incident angle of $82^{\circ}$. The contribution of the detector resolution has a minimum at about $1300 \mathrm{keV}$ due to the stopping power maximum, and increases for higher energies together with the energy-loss straggling contribution. This increase is counterbalanced by the decrease of the multiple-scattering contribution at higher energies together with geometrical straggling, thus resulting in an almost flat energy dependence of the depth resolution for $1300-3000 \mathrm{keV}$. This example shows, that simple rules-of-thumb are usually insufficient to obtain the optimum experimental conditions, and typically all energy broadening 


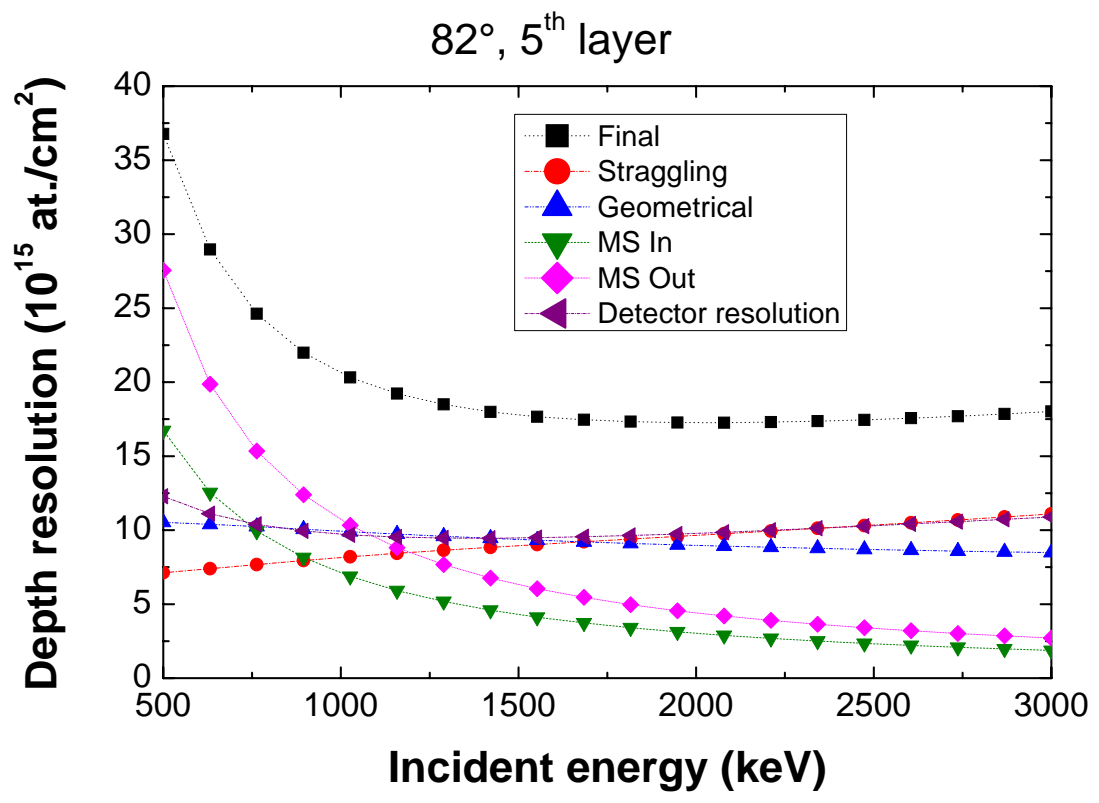

Fig. 3. Energy broadening contributions (transformed to depth resolution) for ${ }^{187} \operatorname{Re}$ in the 5th Re-layer at an incident angle of $82^{\circ}$ as a function of incident energy. Final: Final depth resolution, taking all contributions into account; Straggling: Electronic energy loss straggling; Geometrical: Geometrical straggling; MS In: Multiple scattering on the incident path; MS Out: Multiple scattering on the outgoing path; Detector resolution: Contribution of detector resolution.

contributions have to be taken into account.

The depth resolution for ${ }^{187}$ Re in the $5^{\text {th }}$ Re-layer is shown in Fig. 4 as a function of incident angle for $1000 \mathrm{keV}$ incident energy. The contributions of detector resolution and energy loss straggling decrease with incident angle, while the contributions of multiple scattering and geometrical straggling increase with angle, resulting in an minimum at about $81^{\circ}$.

A possible optimization strategy for this type of samples would be to use an optimum angle/energy combination for each layer. In case of broad optima lower energies are advantageous due to count statistics, and less grazing incident angles due to the possibility to use a wider beam and higher beam currents. It should be also kept in mind that the theoretically possible depth resolution may be not reached in practical applications due to surface roughness. The effects of surface roughness increase with increasing angle, thus favoring less grazing angles of incidence. 


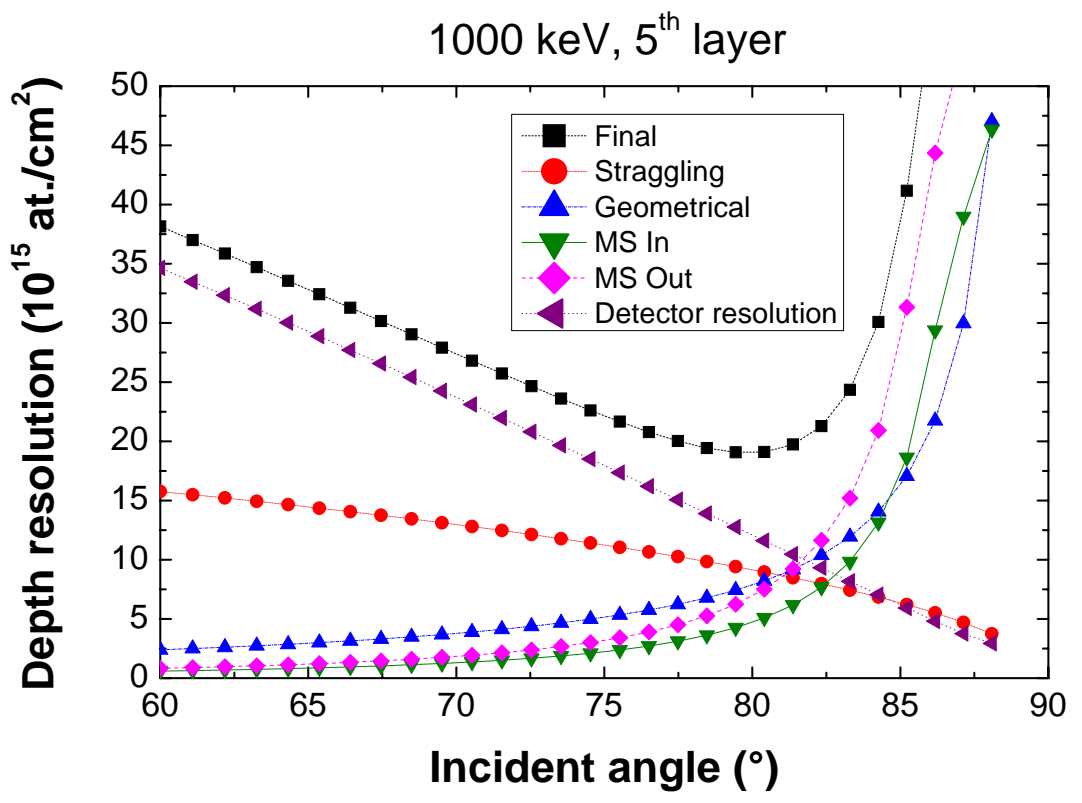

Fig. 4. Energy broadening contributions (transformed to depth resolution) for ${ }^{187} \mathrm{Re}$ in the 5th Re-layer at an incident energy of $1000 \mathrm{keV}$ a function of incident angle. The abbreviations are identical to Fig. 3 .

\section{2 $E R D A$}

ERDA with incident ${ }^{4} \mathrm{He}$ ions and a stopper foil is often used for depth profiling of hydrogen. We consider the example of $\mathrm{H}$ in an amorphous hydrocarbon layer with composition $\mathrm{C}_{0.7} \mathrm{H}_{0.3}$, recoil angle $30^{\circ}, 0.5 \times 1 \mathrm{~mm}^{2}$ incident beam, $0.5 \times 2 \mathrm{~mm}^{2}$ detector aperture at a distance of $50 \mathrm{~mm}, 5.5 \mu \mathrm{m}$ thick Ni-foil in front of the detector. This foil is thick enough to fully stop ${ }^{4} \mathrm{He}$ ions up to energies of about $3200 \mathrm{keV}$.

The depth resolution for a depth of $5 \times 10^{17}$ at $/ \mathrm{cm}^{2}$ (about $50 \mathrm{~nm}$ ) is shown in Fig. 5. For very grazing incident or exit angles (i.e. close to $90^{\circ}$ and $60^{\circ}$ ) the depth resolution deteriorates quickly due to geometrical straggling and multiple scattering on the incident or exit paths. The optimum depth resolution is reached for an incident angle of $61^{\circ}$. This would result in an exit angle of $89^{\circ}$, which is almost impossible to reach in practical applications. The worst depth resolution is obtained for $72^{\circ}$ at all energies: This is very close to the often used symmetric setup with $75^{\circ}$ incident and exit angles. Although the shown result is only valid for the given geometry, target and depth, it should be noted that the symmetric experimental setup with identical incident and exit angles is often close to the worst possible solution. A tilt of the sample towards more grazing incidence or exit angles often provides better results. 


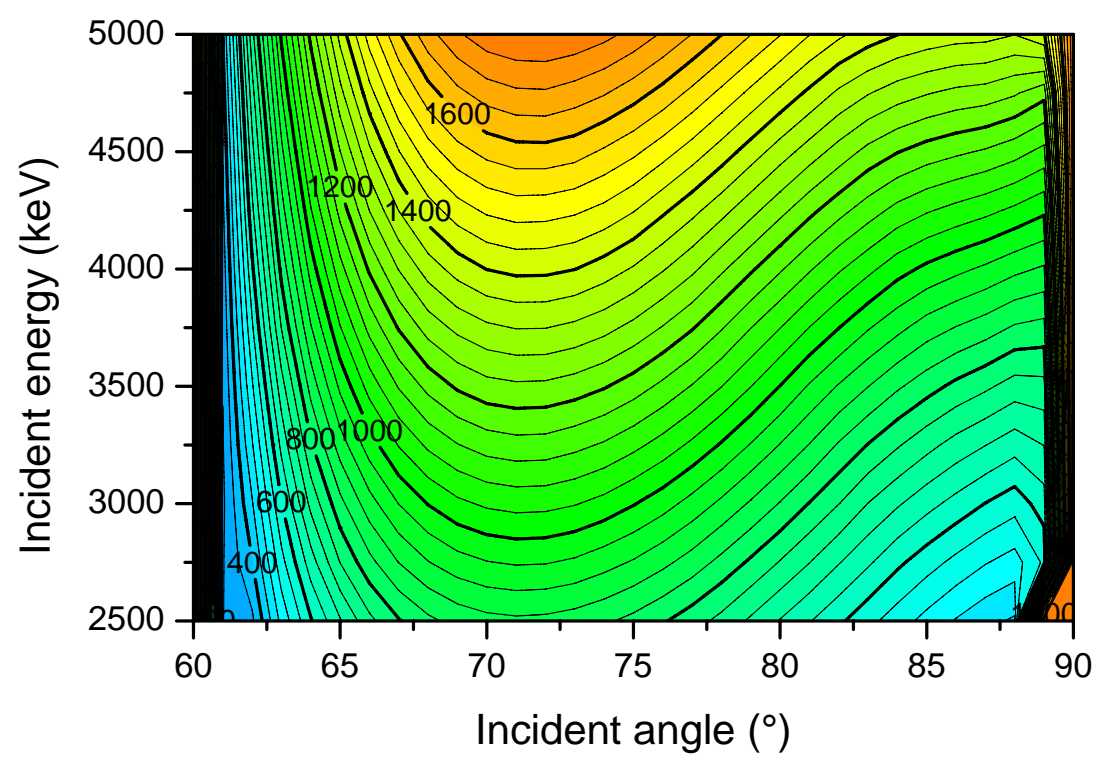

Fig. 5. Depth resolution (in $10^{15}$ atoms $\left./ \mathrm{cm}^{2}\right)$ for ERDA using ${ }^{1} \mathrm{H}\left({ }^{4} \mathrm{He},{ }^{1} \mathrm{H}\right){ }^{4} \mathrm{He}$ in $\mathrm{C}_{0.7} \mathrm{H}_{0.3}$ in a depth of $5 \times 10^{17} \mathrm{at} / \mathrm{cm}^{2}$.

\section{$3.3 N R A$}

Nuclear reaction analysis (NRA) and backscattering with non-Rutherford crosssections often has an additional constraint: The incident projectiles should have a specific energy in the depth of interest, usually due to a maximum in the cross-section. For these cases we have to find the optimum energy/angle combination with the additional constraint, that the projectile energy should have a specific value in a given depth.

As example we consider carbon implanted into tungsten in a depth of $5 \times$ $10^{17}$ at $/ \mathrm{cm}^{2}$, which is detected using the ${ }^{12} \mathrm{C}\left({ }^{3} \mathrm{He}, \mathrm{p}_{1}\right){ }^{14} \mathrm{~N}$ reaction at $165^{\circ}$ scattering angle. This reaction has a maximum in the cross-section at about $2400 \mathrm{keV}$. The depth resolution as function of incident energy and angle is shown in Fig. 6. There is a broad minimum of the depth resolution for incident angles from $72-77^{\circ}$ and energies from $2000-3500 \mathrm{keV}$, providing a resolution of about $1.2 \times 10^{17} \mathrm{at} / \mathrm{cm}^{2}$. The dashed line is the incident energy, which results in a projectile energy of $2400 \mathrm{keV}$ in a depth of $5 \times 10^{17}$ at. $/ \mathrm{cm}^{2}$, i.e. the incident energy which provides the maximum cross-section in the depth of interest. With the additional constraint of maximum cross-section in the depth of interest the optimum depth resolution is obtained for an incident angle of $76^{\circ}$ at an incident energy of $2600 \mathrm{keV}$. 


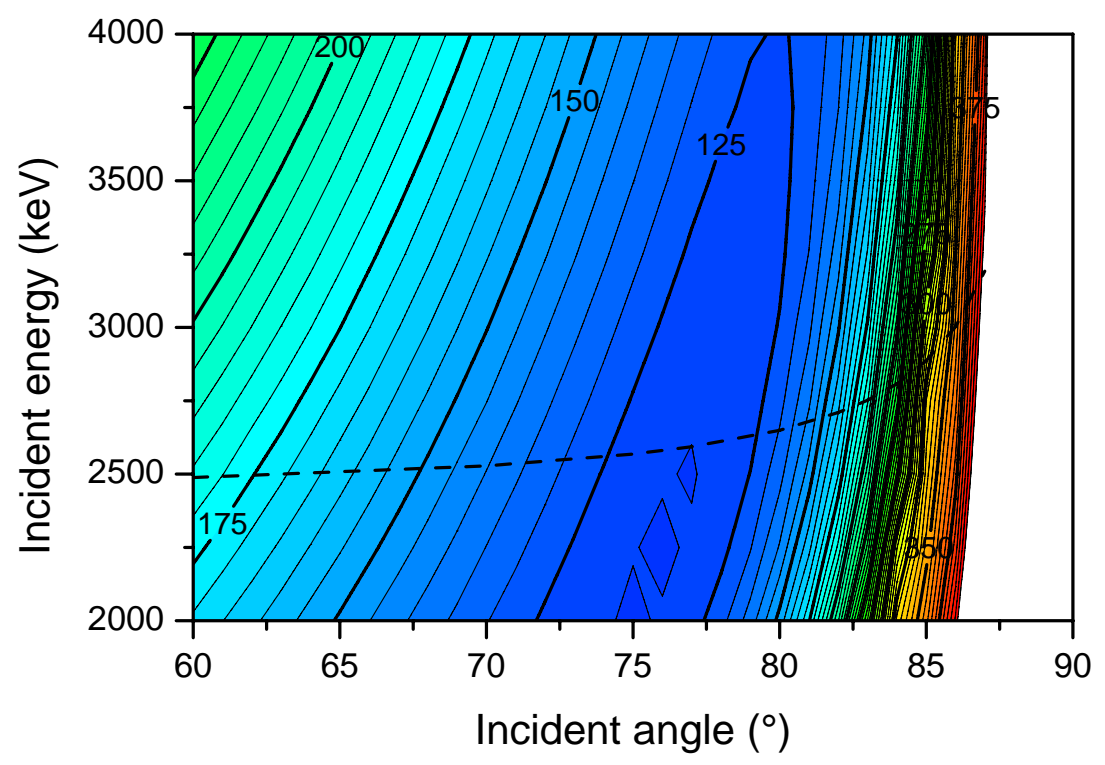

Fig. 6. Depth resolution for the ${ }^{12} \mathrm{C}\left({ }^{3} \mathrm{He}, \mathrm{p}_{1}\right){ }^{14} \mathrm{~N}$ reaction (in $10^{15}$ atoms $/ \mathrm{cm}^{2}$ ) as a function of incident angle and incident energy. ${ }^{12} \mathrm{C}$ is implanted in $\mathrm{W}$ into a depth of $5 \times 10^{17}$ at. $/ \mathrm{cm}^{2}$. Dashed line: Incident energy as function of incident angle, which gives a projectile energy of $2400 \mathrm{keV}$ in a depth of $5 \times 10^{17} \mathrm{at} . / \mathrm{cm}^{2}$.

\subsection{Conclusions}

The RESOLNRA code was developed and allows to optimize the experimental conditions (incident energy and angle) in order to achieve the optimal depth resolution. The code is based on the well established spectrum simulation code SIMNRA. RESOLNRA can be used for RBS, ERDA and NRA and includes the energy broadening contributions due to electronic energy loss straggling, geometrical straggling, multiple scattering, absorber foils and detector resolution. The asymmetry of energy distributions at grazing incidence or exit angles is taken into account for geometrical straggling. Additional constraints (minimum or maximum possible beam energies, limitations of incident or exit angles, requirement for a specific projectile energy in a specific depth) can be taken into account, thus allowing to find the optimum experimental conditions under these additional experimental constraints. The user interface was developed in order to allow a quick optimization of the experimental conditions. The use of identical input files by RESOLNRA and SIMNRA offers the possibility of a rapid experimental design/experimental data evaluation cycle. 


\section{Acknowledgements}

Helpful discussions with E. Szilágyi about the different straggling contributions and with N.P. Barradas about the experimental difficulties at grazing angles are gratefully acknowledged.

\section{References}

[1] E. Szilágyi. Nucl. Instr. Meth. B 161-163 (2000) 37.

[2] E. Szilágyi, F. Pászti, and G. Amsel. Nucl. Instr. Meth. B 100 (1995) 103.

[3] G. Amsel, G. Battistig, and A. L'Hoir. Nucl. Instr. Meth. B 201 (2003) 325.

[4] M. Mayer. SIMNRA user's guide. Tech. Rep. IPP 9/113, Max-Planck-Institut für Plasmaphysik, Garching, 1997.

[5] M. Mayer, K. Arstila, K. Nordlund, E. Edelmann, and J. Keinonen. Nucl. Instr. Meth. B 249 (2006) 823.

[6] M. Mayer. SIMNRA, a simulation program for the analysis of NRA, RBS and ERDA. In Proceedings of the 15th International Conference on the Application of Accelerators in Research and Industry (Woodbury, New York, 1999), J. L. Duggan and I. Morgan, Eds., vol. 475 of AIP Conference Proceedings, American Institute of Physics, p. 541.

[7] W.K. Chu, J.W. Mayer, and M.A. Nicolet. Backscattering Spectrometry. Academic Press, New York, San Francisco, London, 1978.

[8] Q. Yang, D.J. O'Connor, and Z. Wang. Nucl. Instr. Meth. B 61 (1991) 149.

[9] C. Tschalär. Nucl. Instr. Meth. 61 (1968) 141.

[10] C. Tschalär. Nucl. Instr. Meth. 64 (1968) 237.

[11] N.P. Barradas, J.C. Soares, M.F. da Silva, F. Pászti, and E. Szilágyi. Nucl. Instr. Meth. B 94 (1994) 266.

[12] N.P. Barradas. Nucl. Instr. Meth. B 190 (2004) 247.

[13] N.P. Barradas, K. Arstila, G. Battistig, M. Bianconi, N. Dytlewski, C. Jeynes, E. Kótai, G. Lulli, M. Mayer, E. Rauhala, E. Szilágyi, and M. Thompson. Nucl. Instr. Meth. B 262 (2007) 281. 